\title{
Chemical and Physiological Change from Calcium Carbonate to Calcium Phosphate in Skeletal Structures
}

\author{
Dee H. Wu, PhD ${ }^{1 *}$, Caroline Preskitt, $B S^{1}$ and Carolyn Gresham-Fiegel, MPH, MS \\ ${ }^{1}$ Department of Radiological Sciences, University of Oklahoma Health Sciences Center, USA \\ ${ }^{2}$ Veterian Technican School, USA
}

\section{Introduction}

The substrates that comprise skeletal structures have evolved over more than 480 million years. We review chemical and physiological factors that may have driven the evolutionary process from an original calcium carbonate composition of skeletal structures to the calcium phosphate-based bone physiology that we observe in vertebrates today. A review of this topic is not without challenges, however. Many of these changes occurred nearly $\mathbf{4 8 0}$ million years ago, as evidenced by fossils from extinct creatures; these relics, and their correlations to extant organisms, are the only tools we have to develop insight into these changes [1]. In this paper, we discuss factors that may have contributed to the divergence of skeletal constituents to try to gain insights into this intriguing and notable physiological evolution. While the biology of these conditions is ultimately complex and no process can fully account for the millions of years of change, the goal is to propose a model and initiate a dialogue that could lead to, for example, targets for drug delivery, orthopedic treatment, prosthetics, bone pathologies, and other ideas for readers to build upon for future research and investigation. Locomotion and the storage and rapid retrieval of phosphorus, a critical mineral, provided a significant impetus for evolution. This review focuses on the chemical evolution toward $\mathrm{CaPO}_{4}$ from $\mathrm{CaCO}_{3}$ that addressed the need for 1) Storage of energy metabolites in the body and 2) Improved retrieval of these metabolites.

Bone physiology is an important domain of study, particularly when it comes to understanding, controlling, and curing disease. Dissemination of these ideas is vital to appreciate the chemical and biological mechanisms that affect many bone disorders. Such diseases include skeletal dysplasia, bone cancer, osteoporosis, renal osteodystrophy, rickets, osteosarcomas, Paget's disease, and disorders surrounding bone-derived hematopoiesis [2]. Their study could lead to a better understanding of and interest in the biological and chemistry of this topic. For example, phosphate is essential for skeletal mineralization and chronic phosphate deficiency leads to rickets and osteomalacia [3]. In addition, extracellular inorganic phosphate $\left(P_{i}\right)$ regulates the expression of various genes related to proliferation, differentiation, and mineralization [3].
In this review, we provide a scaffold of the morphological and chemical development of phosphate-based bone physiology and focus on the incompletely understood evolution from calcium carbonate to calcium phosphate in skeletal structures.

At first glance, calcium phosphate may appear to be disadvantageous in organisms, as it possesses a higher molecular weight than that of calcium carbonate. One report notes that without the continuous animal domain participating in the phosphorus cycle, catastrophic depletion of phosphorus exchange in the cycle may occur [4]. Phosphorus is the only element without a significant gaseous and soluble phase. As such, it cycles through the environment much more slowly than nitrogen or carbon. Thus, its movement is primarily driven by tectonic processes [4]. Why then would organisms select to incur the additional weight and lack of bioavailability of phosphate? The answer is likely due to the dynamic nature of calcium phosphate and its ability to support various physiological processes, including storage of metabolites, retrieval of metabolites for energy and locomotion, protection against skeletal degradation, and skeletal remodeling in response to mechanical stress.

\section{Setting the Stage in the Cambrian Era}

Early life forms developed an exoskeleton based on calcium carbonate, indicating a divergence involving phosphate as opposed to calcite in vertebrate bone. This physiological divergence poses the question of why the osseous matter of

*Corresponding author: Dee $\mathrm{H} \mathrm{Wu}, \mathrm{PhD}$, Chief, Technology Applications \& Translational Research, Associate Professor, Department of Radiological Sciences, University of Oklahoma Health Sciences Center, Garrison Room 3258, 940 NE $13^{\text {th }}$ Street, Stanton L. Young, Oklahoma City, OK 73104, USA, Tel: 405-271-8001 x 35405 (Lab); 405-271-8001 x 46993 (Office); Fax: (405)-271-3462

Accepted: January 21, 2021

Published online: January 23, 2021

Citation: Wu DH, Preskitt C, Gresham-Fiegel C (2021) Chemical and Physiological Change from Calcium Carbonate to Calcium Phosphate in Skeletal Structures. Insights Biomed Res 5(1):139148 
Citation: Wu DH, Preskitt C, Gresham-Fiegel C (2021) Chemical and Physiological Change from Calcium Carbonate to Calcium Phosphate in Skeletal Structures. Insights Biomed Res 5(1):139-148

early aquatic lifeforms required calcium carbonate and, if calcite-based bone physiology had a selective advantage, why a shift to a phosphate-based system occurred.

The first invertebrates developed in the oceans 488-543 million years ago during the Cambrian era [1]. In this period, primarily soft-bodied animals with an external shell or carapace existed [5]. The primary component of the shells of these early aquatic lifeforms was calcium carbonate, a structurally strong material that persists for extensive periods. Indeed, the shells of these antediluvian creatures lasted without change for tens of millions of years. This is evidenced by the presence of limestone (the calcite- and aragonite-containing mineral form of calcium carbonate), which is formed through the sedimentation of skeletal fragments of marine organisms such as coral, foraminifera, and mollusks [6]. Calcium carbonate may have been a perfect starting component for the outer structure of invertebrate marine creatures. The initial presence of calcium carbonate may have arisen from the turbulent movement of tectonic plates 1.5 billion years ago, which deposited copious amounts of minerals into the oceans [7]. The abundance of calcitic minerals and intense environmental conditions may have contributed to the rapid increase in the diversity of multicellular organisms (a period referred to as the Cambrian explosion), as organisms may have been required to develop calcite exoskeletons for protection, as well as other calcite-containing structures for predation, locomotion, or competition [7]. To attempt to better understand the evolution of skeletal structures, it is informative to examine each of the individual elemental constituents that construct the basic chemical building blocks within calcium carbonate and calcium phosphate.

\section{Constituents of Calcium Carbonate and Calci- um Phosphate}

Calcium, specifically $\mathrm{Ca}^{2+}$, plays a significant role in eukaryotic biology. It is critically involved in cell signaling and requires essential homeostasis control. This is achieved through regulation of extracellular and intracellular ion concentrations [1]. The need for calcium in the regulation of cellular processes suggests a need for the development of absorptive structures, such as gills, which allow for gaseous exchange and permeation of ions involved in the growth and development of osseous structures. For example, early exoskeleton-containing life forms developed ctenidia, a respiratory organ or gill, which is still observed in many modern mollusks. This structure also exists in bivalves, cephalopods, polyplacophorans (chitons), and aquatic gastropods, such as freshwater snails and marine snails [8]. Shell formation in these organisms necessitates an increase in the uptake of calcium ions from surrounding seawater and movement of these ions from the hemolymph (blood equivalent in invertebrates) to extrapallial fluid (extracellular fluid equivalent in invertebrates), where calcification and subsequent extracellular secretion occur [9]. The ctenidium (gill) of the giant clam, Tridacna squamosa, is observed to contain voltage-gated calcium channels that regulate calcium concentration gradients, demonstrating that the gill plays a role in acquiring chemical constituents from the environment and ultimately forming an exoskeleton [9]. Similarly, calcium plays a role in the mineralization of osseous structures in vertebrates, such as bone and antlers, which we will discuss in a later section [1].

Carbon, considered to be fundamental to organic lifeforms, was likely available in abundance from volcanic and coal components in the Earth [10]. Unique in comparison to all of the elements, carbon is the only element able to form chains of thousands of atoms as well as ring structures [10]. Due to its ability to form large, strong, and highly stable structures, carbon acts as the central atom to which nonmetals can bind, such as the case of carbonates. The stability and hardness that carbon structures impart are important to the constituent nature of the 'skeleton' that is used to frame an organism's body. Bioavailability also serves an important role. Thus, carbon was requisite to the formation of the carbonate exoskeleton.

Oxygen is essential for respiration in all living organisms and plays a central role in cellular processes, including the formation of osseous structures [10]. Respiration is the foundation of metabolic processes, which require the exchange of gas between the internal and external environments. Working in tandem with carbon to form carbon monoxide (a byproduct of cellular processes like metabolism), oxygen enables growth, development, and survival. Hsia, et al. provide that all cellular systems, including the skeletal system, are "adapted to deliver enough oxygen and eliminate enough carbon dioxide to allow the species to surmount specific environmental and predatory pressures while simultaneously limiting the energy cost of breathing and cumulative oxidative stress in cells and organelles within an acceptable range" [11]. Interestingly, the oxygen isotope concentration in the shells of mollusks and other early life forms is dependent on the temperature and salinity of the external environment [12]. This substantiates the idea that the exoskeletons of organisms formed in response to environmental pressures through an internal oxygen-mediated pathway. While carbon is only contained in calcium carbonate, oxygen is present in both calcium carbonate and calcium phosphate, making up $48 \%$ and $41 \%$ of their molecular masses, respectively.

Phosphorus primarily exists in nature in mineral forms such as apatites, or oxygen- and calcium-containing groups, due to its resonance and reactivity [13]. This reactivity enables the process of phosphorylation and dephosphorylation, which are necessary in carrying out active cellular processes. Due to its affinity to form chains with other phosphorus molecules, it is the primary component in chain-like structures, such as DNA and RNA, as well as adenosine triphosphate (ATP) [13]. The bioavailability of phosphorus is dependent on the phosphorus cycle, which describes the movement of phosphorus through the ecological biosphere. Phosphorus is a limiting nutrient to aquatic organisms and is not readily released into the atmosphere; however, phosphorus can be found in rocks and minerals [4]. Therefore, the weathering of rocks and minerals releases phosphorus into the environment at which point it is available for organisms to uptake.

\section{Collagen and Mineralization}

Collagen is the primary matrix protein in tissues and 


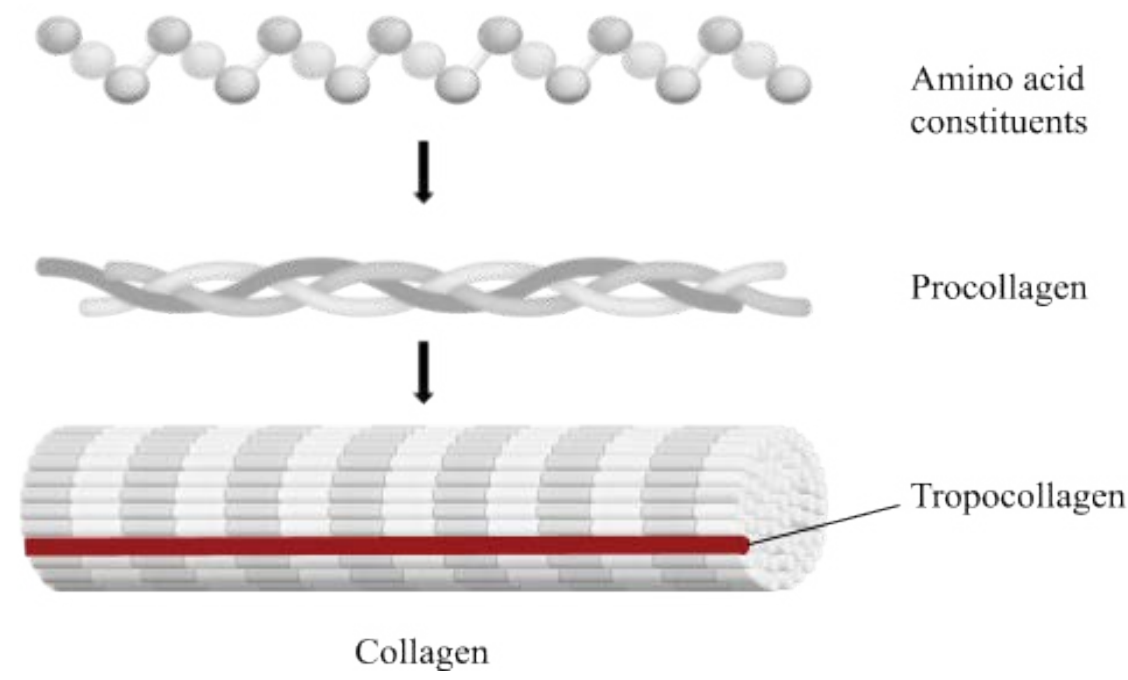

Figure 1: Collagen and its precursors: The top illustration shows amino-acid residues which combine to form procollagen (including noncollagen end terminal peptides) shown in the middle illustration. Procollagen then forms tropocollagen through disulphide crosslinking. Depending on degree of crosslinking, or maturation, the tropocollagen forms collagen fibrils, shown in the bottom illustration [15].

skeletal structures. Collagen likely first appeared in the Proteozoic era and diversified into 1) A fibril-forming class of collagen used in the stiffening of tissues and bones, and 2) A non-fibril-forming class of collagen that formed the basement membranes of structures $[14,15]$. The collagen of the matrix consists of fibers in strands of repeating units that are crosslinked and provides bone with its tensile strength. These fibers are formed from collagenous precursors procollagen and tropocollagen. Procollagen contains peptide tails that maintain central amino-acid residues until secretion, while tropocollagen forms triple helices that cross-link via hydrogen disulphide bridges with other tropocollagen to form mature collagen fibrils (Figure 1) [15]. Studies comparing collagen composition in vertebrates and invertebrates have attempted to categorize collagenous structures on the basis of differing amino acid concentrations [14]. While vertebrate collagen composition is similar across species, invertebrate collagen composition is highly variable, suggesting that the needs of collagen-containing structures require individual modification [14]. Because modern vertebrates rely on a calcium-phosphate based skeletal physiology, the ability for collagen to form the basement membranes of organs as well as to provide strength and elasticity of vessels, tissues, and bones is likely highly conserved. Moreover, invertebrates comprise more than $95 \%$ of known species, some containing calcium-carbonate based exoskeletons, so there is an inherent need for diversified collagen function and composition among invertebrate species [16].

Collagen plays an essential role in the development of active structures and substrates that are essential for locomotion. Collagen accounts for the elasticity of bone to withstand mechanical stress, and it serves as the nucleation site of hydroxyapatite crystals stored between tropocollagen molecules through a complex biological cross-linking process [17] Collagen is also necessary for the formation of vascular structures, body tissue, and cartilage. Endochondral ossification is the process in which cartilage is mineralized to form bone, which provides evidence that collagen was already present prior to integration of calcium phosphate in skeletal systems and evolved to reflect individual tissue requirements of developing phosphate-based osseous structures $[7,14]$.

\section{Osteoclast Resorption and Osteoblast Re- modeling}

Osteocytes are intimately involved in mineral homeostasis, both through local resorption and reforming of the matrix surrounding them and by the release of fibroblast growth factor-23, which reduces renal reabsorption of phosphate [18]. Adequate phosphate is critical for apoptosis (programmed cell death) of mature chondrocytes in the growth plate [18]. Lack of adequate phosphate results in decreased apoptosis and blocked bone growth [18].

Acellular, or anosteocytic, refers to the absence of enclosed osteocytes within the extracellular matrix of the bone [19]. Many bony fish species which exist today (such as Teleosts) are acellular, however, all terrestrial vertebrates require cellular bone [1]. Teleosts descended from cellular bone-containing organisms which suggest there was an initial need for development of active bone remodeling within vertebrates and that divergence into acellular bone occurred later [19]. Teleosts still possess the ability to differentiate surface osteocytes into extracellular osteocytes for purposes of remodeling, though that mechanism of differentiation is beyond the scope of this review.

Cellular bone is composed of four different cell types: osteoblasts, osteoclasts, bone lining cells, and osteocytes (Figure 2). Osteoblasts, osteoclasts, and bone lining cells are derived from mesenchymal (progenitor) cells and are contained on the surface of the bone, whereas osteocytes, an enclosed form of osteoblasts, exist within the lacunae (pores) of the bone [20]. Osteoblasts promote mineralization of the surface of the bone and act as nucleation sites for the formation of hydroxyapatite through accumulation of calcium and phos- 


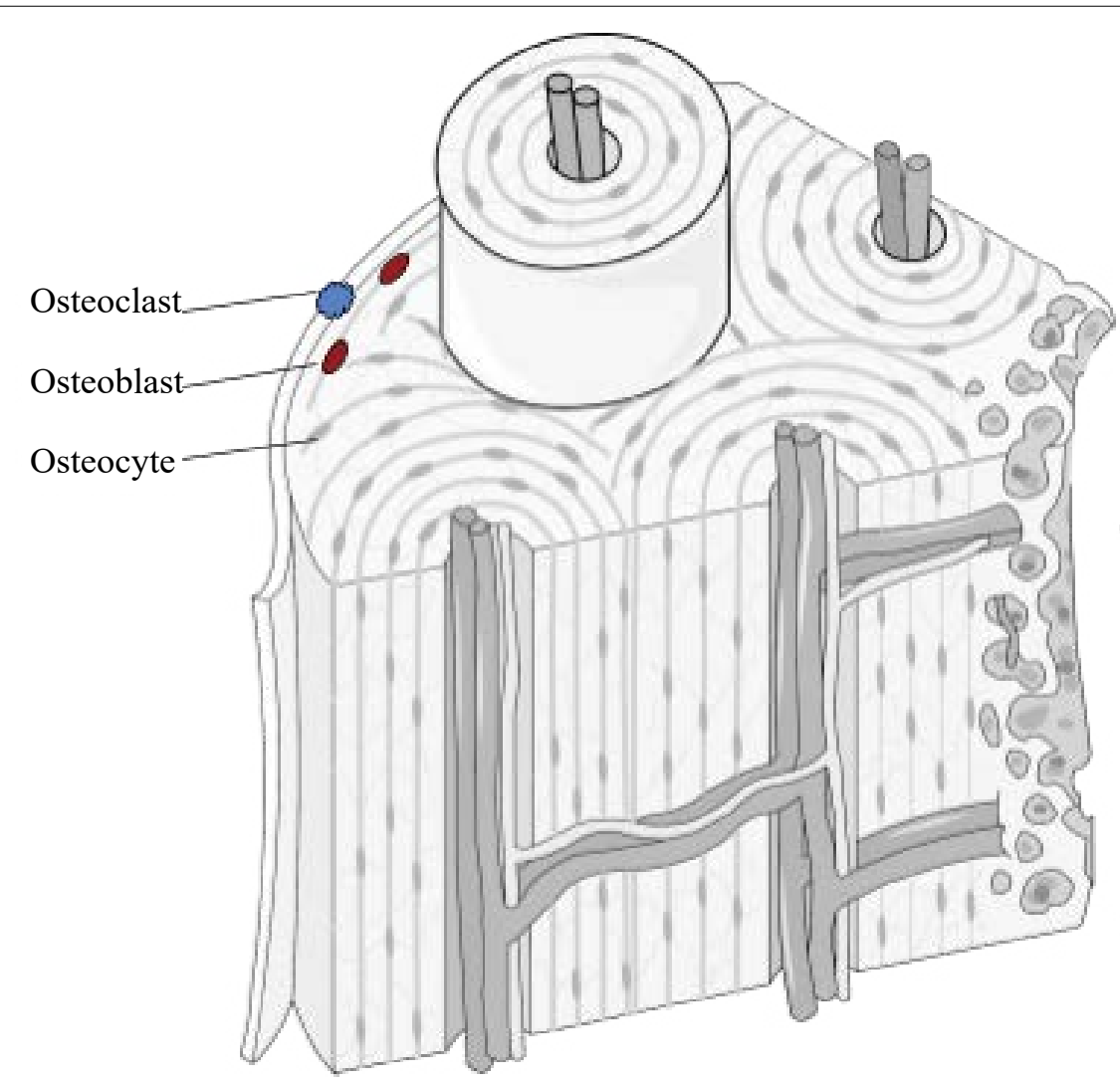

Figure 2: Internal structure of bone: The osteocytes are pictured immediately surrounding the blood vessels and nerves, enclosed in the matrix of the bone. Osteoclasts and osteoblasts are pictured in blue and red, resting on the surface of the bone.

phate molecules [20]. Osteocytes play the same role in bone remodeling and mineralization, though they are the more mature differentiation state of osteoblasts and can exist in both active and inactive phases [20]. Conversely, osteoclasts are phagocytic cells that resorb bone in response to external stress and environment. Both the osteoblast and osteoclast maintain an equilibrium of the amount of bone resorbed versus bone mineralized through a coupling mechanism [20].

The calcium carbonate-based shells of mollusks, such as snails, clams, and oysters, are acellular. Thus, they do not undergo remodeling and grow in a bottom-up process through the addition of new material to the margins of the existing shell [21]. Rather, mantle tissue located under the shell secretes proteins extracellularly to form a structural basis with a mineral overlay. While this may be effective for stationary exoskeleton-containing species, organisms containing an endoskeleton, and which utilize locomotion require a mechanism of bone remodeling to respond to ongoing mechanical stresses, which a phosphate-based system allows.

Even among vertebrate species, however, there are many exceptions to the "rules" of skeletal structure, including the anosteocytic bone of many teleost fish species, the different types of lamellar bone in tetrapods, bone-cartilage intermediates such as chondroid bone, and hyper-ossified otic bone tissue [22]. However, all of these bone tissues are unified in their basic composition. The organic component of bone is primarily a honeycomb-like matrix material, made of more than 90\% Type I collagen and the remainder non-collagenous proteins [20]. Even in species lacking osteocytes, their osse- ous structures still contain mesenchymal-derived osteoblasts and hematopoietic-derived osteoclasts used for bone remodeling [22].

\section{Bone and Calcium Phosphate}

Vertebrate bone is primarily composed of calcium phosphate in the form of hydroxyapatite, which in conjunction with type I collagen and proteoglycans forms a structure that supports a multitude of functions, including internal organ protection, muscle anchoring, metabolite storage, and growth and repair [23]. This illustrates the fact that bone is a dynamic, facile and metabolically active organ requiring a more complex biochemical substrate. Evolutionary changes could have stimulated this divergence to incorporate a more dynamic and agile chemical composition in the response to environmental and organismal changes [1]. It is useful to bear in mind the complexity of an organism's adaptations, which can include the environment, bioavailability of materials, and competition between and within developing species. These factors could have been the impetus that evoked physiological change.

During the development of Ostracoderms (early vertebrates) of the Paleozoic era, phosphate and calcite presumably were equally available in the marine environment, as there is no present evidence that marine phosphate concentrations were different from our present era [24]. However, one of the primary hypotheses supporting the divergence from calcium carbonate to calcium phosphate based on marine mineral concentration is Paleozoic overabundance 
of phosphorus [8]. After a period in which organisms of uncertain phyla used both calcium phosphate and calcium carbonate, speciation on the basis of preferred mineral likely occurred during the Ordovician era [25]. Consequently, invertebrates moved toward exoskeletons comprised of calcium carbonate, while vertebrates moved toward an endoskeleton comprised of calcium phosphate, suggesting a parallel evolutionary trend.

\section{Chemical and Structural Differences between Calcium Carbonate and Calcium Phosphate}

At this point, it is informative to look more deeply at the differences between the chemical makeup of the constituent matter of phosphate and calcite-based skeletal structures (Figure 3).

Calcium carbonate is an ionic solid, composed of carbon, oxygen, and an ionically bonded calcium di-cation, as previously discussed. It is relatively insoluble in water, except in environments of salt water, in which it dissolves into its constituent polymorph forms of calcite and aragonite through a dissolution-precipitation process, though other polymorph forms exist [26]. The crystallinity of calcium carbonate, or the ability to dissociate into its constituent ions, is highly dependent on the $\mathrm{pH}$ of the solution in which it is precipitated, as well as the kinetics of the environment (constant agitation of constituent molecules through movement of ocean water) [27]. This suggests that calcium carbonate may have not only played a role in strength of osseous structures, but also allowed for molting of external structures through easy dissolution [28]. Amorphous calcium carbonate, the precursor of more crystalline polymorphs, is relatively unstable; thus, it favors a more crystalline and less porous state due to the tightly compacted organization of its individual mineral grains (Figure 4) [29]. This also demonstrates a requirement for more stable molecules to be incorporated into bone and skeletal physiological processes, as organisms that utilize amorphous

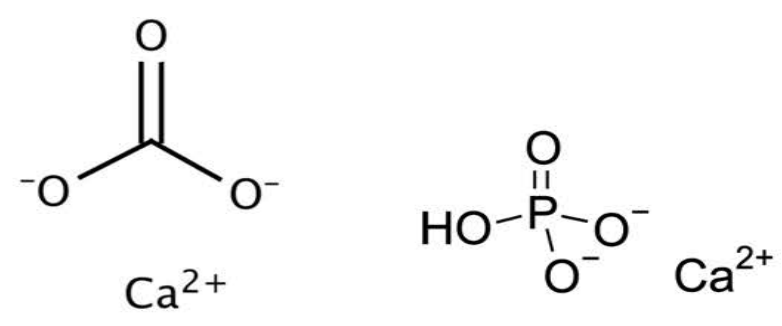

Figure 3: Chemical structures of $\mathrm{CaCO}_{3}$ and $\mathrm{CaPO}_{4}$. calcium carbonate also contain proteins and other molecules that stabilize the reactive polymorph [29]. Because carbonate-based exoskeletons do not undergo remodeling, shells are often comprised of three distinct layers, each differing in their concentration of minerals and protein: an outer uncalcified protein layer, a middle calcite prismatic layer, and an inner aragonite layer of pearly nacre [30].

Likewise, calcium phosphate is an ionic solid composed of calcium, phosphorus, and oxygen, though it most commonly exists in the mineral form of hydroxyapatite. Hydroxyapatite is the primary inorganic component in bone and tooth enamel and is characterized by very small crystals $(50 \times 25 \times 5 \mathrm{~nm})$, low hydroxide content, and poor crystallinity [31]. Its relatively low degree of crystallinity contributes to its increased porosity, as it organizes into an amorphous configuration and has greater distance between individual mineral grains (Figure 4). It is insoluble in water and easy to precipitate, which is necessary in the process of mineralization. However, the $\mathrm{pH}$ and degree of saturation within acidic environments affect solubility. While pure hydroxyapatite has a calcium to phosphate ratio of 5:3 (1.67), bone mineral itself has calcium to phosphate ratios ranging from $1.37-1.87$. The complete composition of bone mineral is complex and contains additional ions, such as silicon, carbonate and zinc, which allow it to stabilize and maintain phosphate homeostasis in the body [23]. Carbonate specifically may account for as much as $5-6 \%$ of hydroxyapatite by weight, which provides additional protection from acidic degradation of osseous structures [23,32]. The ability of calcium phosphate to form graded structures of varying strengths and elasticity makes phosphate a selectively favorable biomineral.

Calcium Carbonate has a molecular weight around $100 \mathrm{~g} /$ $\mathrm{mol}$ and density of $2.8 \mathrm{~g} / \mathrm{cm}$, while calcium phosphate has a molecular weight of $310 \mathrm{~g} / \mathrm{mol}$ and a density of $3.14 \mathrm{~g} / \mathrm{cm}$. If the molecular weight of calcium phosphate is much greater than that of calcium carbonate, why then are the densities similar? This is due to the difference in porosity of both compounds. Because calcium phosphate prefers an amorphous structure, it contains greater space between intermolecular junctions. Porosity is defined as the ratio of pore space in the mineral and is determined by pore space divided by the total volume, given by the formula below [33].

$$
n=\frac{V_{\text {pore space }}}{V_{\text {total }}}
$$

Increased porosity of calcium phosphate makes it selectively advantageous for osseous structures, as it still has rel-

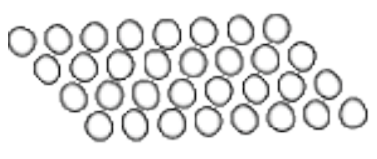

Crystalline

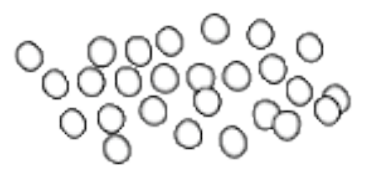

Semi-Crystalline

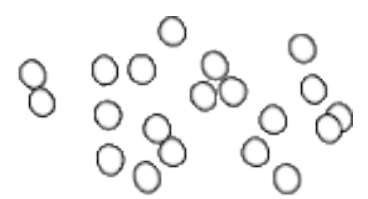

Amorphous

Figure 4: Illustration of the organization of individual mineral grains in crystalline, semi-crystalline, and amorphous (or porous) minerals. 
atively low density despite phosphorus being a 'heavier' element. Additionally, the porosity allows for more elasticity and therefore strength for locomotion, greater vascularity within the bone to promote metabolite exchange, and increased surface area to decrease lactic acid saturation.

\section{Locomotion, a Primary Driver for Change}

While the ability to excrete a rigid outer skeleton certainly promoted survival by protecting organisms from predation and mechanical damage, an endoskeleton fosters more rapid locomotion for escape and food gathering through the development of longer and stronger limbs [34,35]. Increased competition for food and the need for safety among ancient organisms may have driven this early skeletal divergence which favored increased movement. However, even mollusks such as clams can provide some locomotion, as they are able to move their bodies to bury themselves in the sand [36]. In order to progress in more efficient and advanced locomotion, the development, hardening, and reshaping of the notochord was necessary [37]. The tail-like structure and ability of muscle to attach to dorsal structures provided the hydraulics that permitted greater locomotion [37]. Because exoskeletons are growth- and locomotor-limiting, the development of notochord-containing endoskeletons allowed organisms to grow larger, more muscular, and enabled them to move faster. This does not come without cost, however, as this also requires more energy and metabolites, as well as increased tensile strength of skeletal structures to support locomotor mechanical stress.

The strength of bone depends on its organic and inorganic components, the interactions between these components, and its degree of mineralization. A number of absorption forces, like hydrogen bonding, van der Waals' interactions, and hydrophobic interactions, confer strength to the collagen-mineral binding [31]. Since phosphates have more hydrogen bond acceptors, the incorporation of phosphate in vertebrate bone results in stronger skeletal structures. Such structures could then provide weight-bearing capability for increasingly large and diverse terrestrial animals. Terrestrial animals have limited intake of calcium and phosphate through diet, whereas aquatic organisms obtain it from surrounding water, so minerals that are acquired must be conserved [4]. Thus, an advanced storage system for phosphates also became necessary for accessing ATP and other metabolites.

Protovertebrates with developing endoskeletons and musculature most likely developed the anaerobic glycolytic system of energy production for intense activity, resulting in ATP generation and consequently formation of lactic acid (Figure 5) [38]. This metabolic pathway enabled early vertebrates to attain levels of burst energy that would not be possible by relying completely on aerobic metabolism [39].

\section{Vascularity and Porosity Allows for Retrieval of Phosphates}

Vascularity allows for active bone growth and remodeling, both in development and in healing. In response to changing mechanical loads, osteocytes express membrane-bound proteins and release soluble factors that manage the activity of

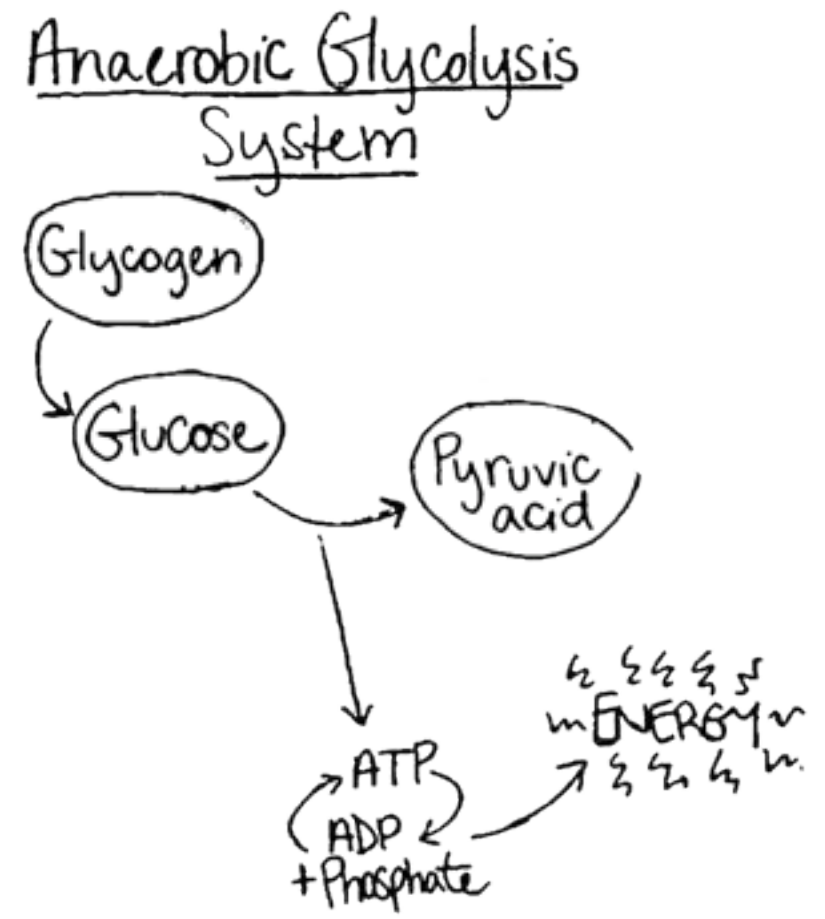

Figure 5: Anaerobic glycolysis pathway. Glycogen is converted resulting in ATP which is used for energy and therefore enables locomotion.

osteoclasts and osteoblasts, to resorb and form bone, respectively [25]. The porosity of hydroxyapatite-containing skeletal structures enables osteocytes to move throughout the canaliculi (spaces in bones) and allows for the diffusion and transport of molecules, cells manufactured in hematopoietic processes, and nutrients between the inside and outside of the bone. Higher porosity is also thought to enhance osteogenesis, as various studies have shown that larger surface area of bone results in higher ion exchange and bone-inducing factor adsorption [40].

Because the density of the molecules comprising the skeleton can add substantial weight to the overall structure, the higher porosity of calcium phosphate is advantageous and the distribution of varying pore sizes throughout the bone contributes to compressive strength of bone [41]. Porosity not only helps with reducing the overall weight, but perhaps provides greater access to the phosphates stored within the bone. Retrieval of the phosphate must be completed in a timely and efficient manner so that the organism can respond quickly to the energetic demands of the competition and environment of its surroundings. Greater porosity and additional interstitial space provide access to small arterioles, venules, and capillaries, which improve adequate diffusion of nutrients and materials to the bone structure, as well as improved phosphorus reuptake and reabsorption (Figure 6) [41].

Endochondral ossification is also intimately tied to vascularization, as blood vessels invade cartilage along migration cavities of chondrocytes [42]. The bones of terrestrial vertebrates show great regularity of structure, long survival of the 

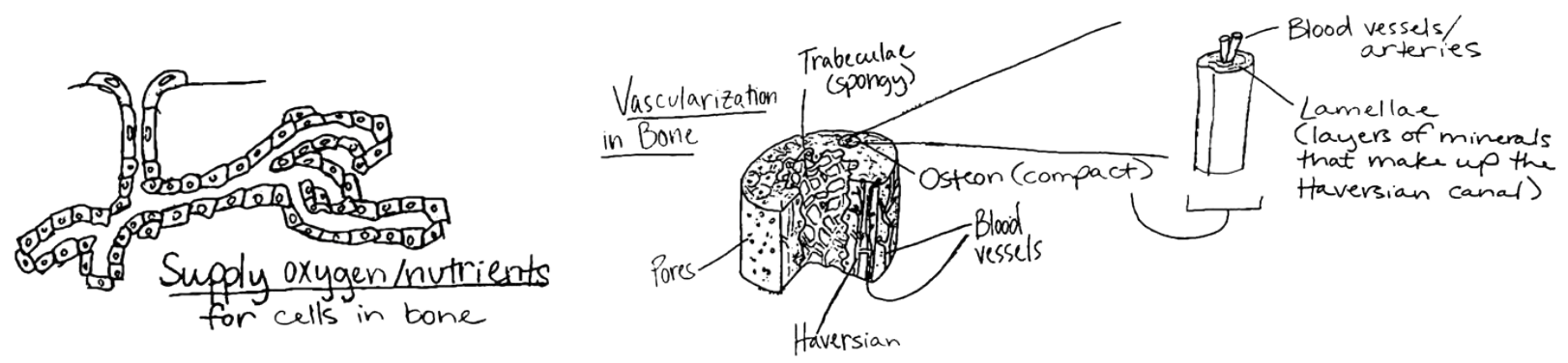

Blood vessels take up and deposit calcium and phosphate for bone remodeling and for mineral storage.

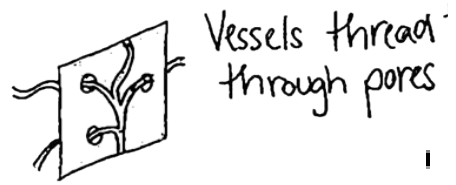

The pores in the bone create more surface area for blood vessels to thread through.

Figure 6: Vascularity and bone porosity allow for efficient diffusion of ions and minerals, osteoblast and osteoclast remodeling, migration of osteocytes and chondrocytes (in the case of endochondral ossification), and increased surface area for storage, exchange, and reduction of lactic acid saturation [40].
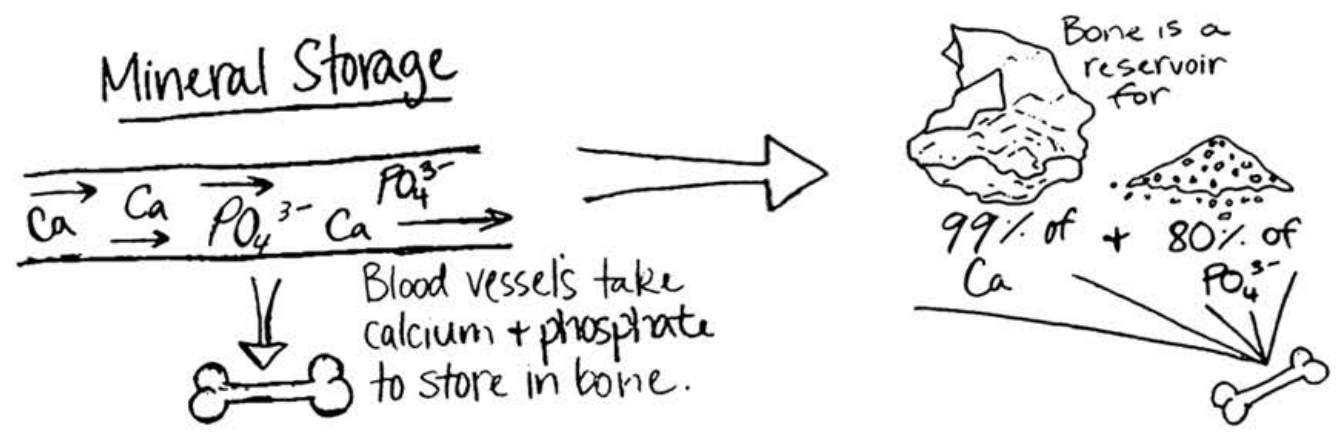

Figure 7: The bone acts as a reservoir for minerals, specifically hydroxyapatite which is stored between tropocollagen helices [15,43].

osteocytes, and nutrient canals and secondary bone arranged in Haversian systems [43]. This vascularized structure lends itself to several functions essential to vertebrate life. Vascular bone serves as a reservoir for phosphate and calcium, with $99 \%$ of the body's calcium and $80 \%$ of its phosphate found in the skeletal system (Figure 7) [44]. The vascular and porous structure renders the minerals more readily available for all functional activities, including muscular contraction and energy production.

\section{Lactic Acid and Osseous Dissolution}

Systemic release of lactic acid spurring from muscular activity results in a reduction in the $\mathrm{pH}$ of extracellular fluids (ECF) within vertebrates [39]. In vertebrates with calcium phosphate-based skeletons, a lactate-induced acidic ECF environment produces only slight skeletal dissolution and few invertebrates show an activity-related increase in acidity $[39,45]$. In fact, some invertebrates use a cytosolic malate dehydrogenase instead of the vertebrate lactate dehydrogenase pathway, thereby circumventing entirely the production of lactate [34]. In organisms which do utilize a lactate dehydrogenase pathway, lactic acid dissolves calcium phosphate and calcium carbonate quite readily due to its $\mathrm{pH}$ of 3.5 [39]. Hydroxyapatite however is able to resist dissolution due to buffering components which it contains and is much more resistant to dissolution than is calcite (Figure 8) $[7,17,39]$. The degree of saturation of lactic acid also contributes to how readily bone is dissolved, hence the need for increased surface area of bone [46].

Studies show that the magnitude of bone dissolution would be significantly greater if the skeleton were calcite-based rather than phosphate-based, demonstrated by a series of in vivo experiments with exercised fish in which the dissolution of implanted calcite was significantly greater than that of implanted hydroxyapatite [39]. Ruben and Bennett concluded that a skeleton based on calcium carbonate would be chronically prone to dissolution even under sub-maximal activity and might be "hazardously soluble after bouts of intense activity" [39]. This may suggest that as Paleozoic vertebrates became increasingly active, their ability to generate ATP anaerobically increased. This modification may have been accompanied by the gradual formation of a more stable, phosphatic skeleton. 

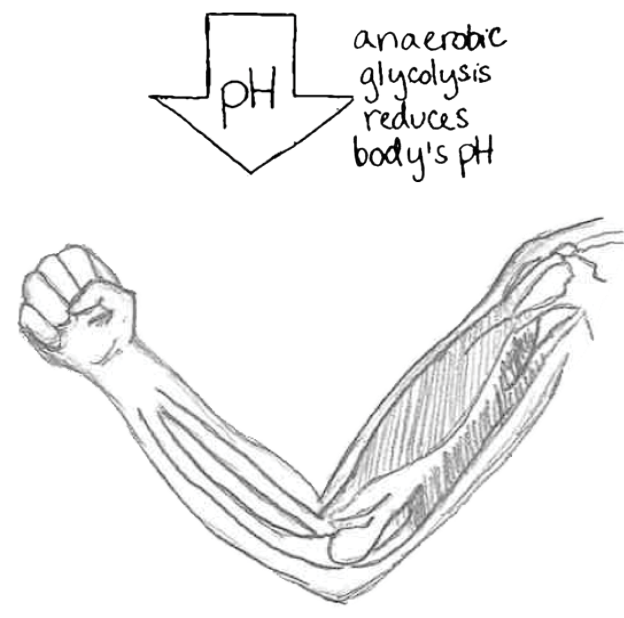

Figure 8: Glycolysis which produces ATP for energy also produces lactic acid as a byproduct. Calcium phosphate is more resistant to dissolution in acidic environments than is calcium carbonate.

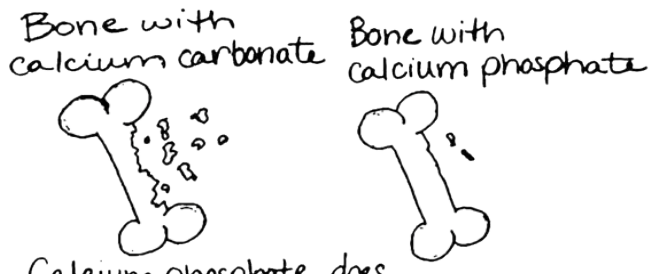

Calcium phosphate does not dissolve as easily

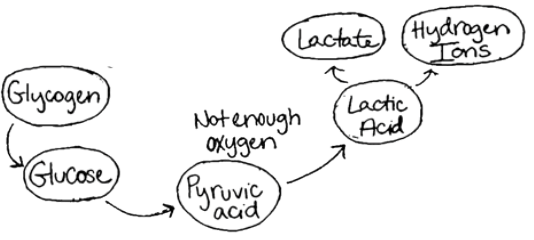

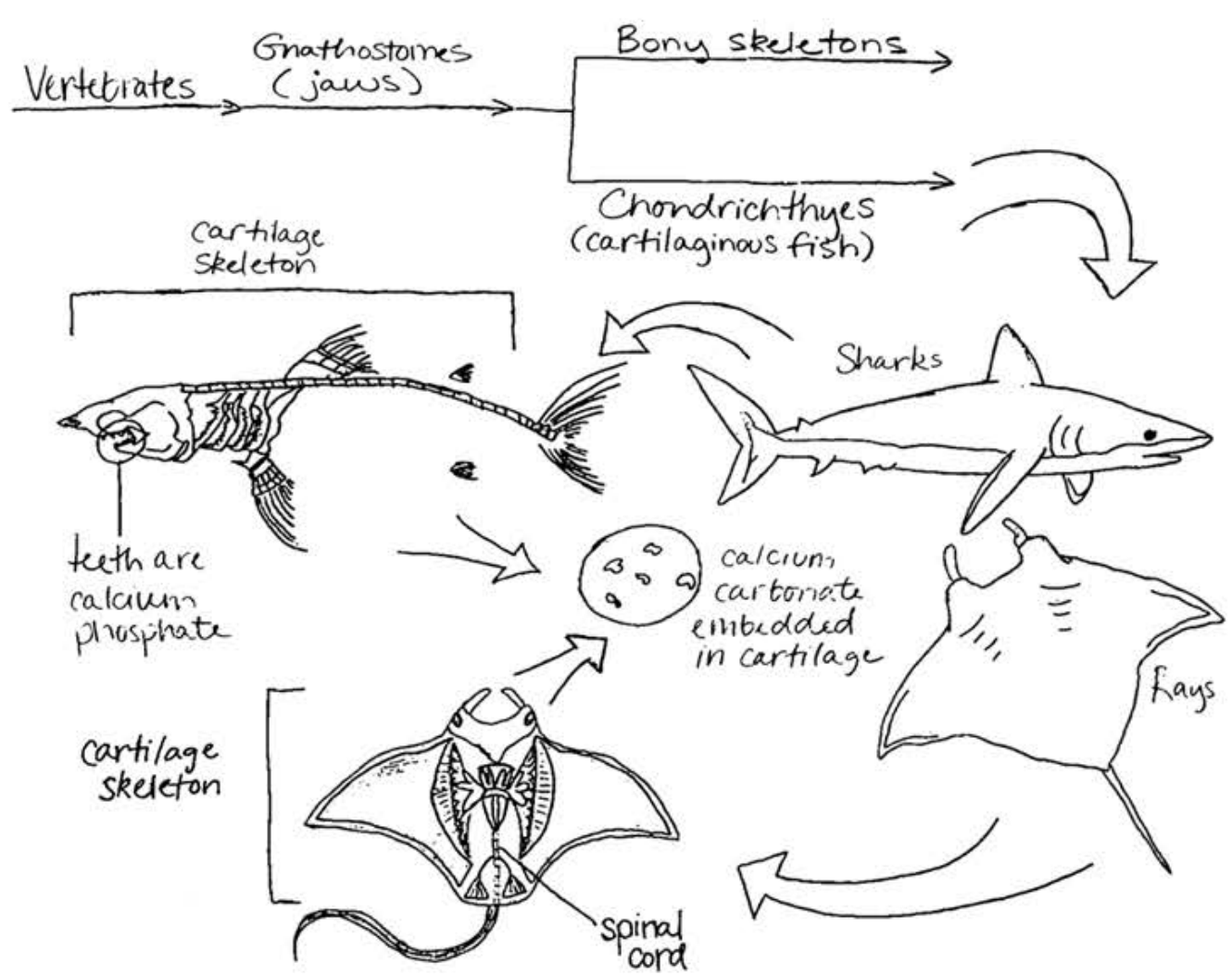

Figure 9: Sharks have teeth made of calcium phosphate and a collagenous cartilage skeleton. However, their skin is made of dentine and is rough to the touch, providing evidence that calcium phosphate was likely acquired to form hard enamel-like structures for feeding and that bony exoskeletons likely dissolved into dentine-like scales [49].

\section{Teeth, Jaws, and Armor}

The mineralized tissue of early (Precambrian) Conodonts suggests that mineralized tissue first appeared in the form of teeth [1]. From the perspective of the cranium, it is possible that emerging creatures required teeth for greater ability to penetrate shells for predation. The development of teeth and various oral apparati was possible due to the ability of different cell subsets, such as enclosed osteocytes versus surface osteocytes, to undergo epithelial-mesenchymal transformation. This ability of cells to transform likely led to diversification and advancement in the teeth and jaw structures within organisms to better respond to their environment.

Simultaneously, mineralized tissue also appeared in the endoskeletons of Galeaspids, which were the precursors of osteocyte-containing mineralized skeletal structures in Ostracoderms [1]. Ostracoderms were heavily armored, fish-like 
Citation: Wu DH, Preskitt C, Gresham-Fiegel C (2021) Chemical and Physiological Change from Calcium Carbonate to Calcium Phosphate in Skeletal Structures. Insights Biomed Res 5(1):139-148

creatures with dermally excreted phosphate-based exoskeletons [8]. The exoskeleton consisted of three layers, a thin outer layer of hard dentine, a middle layer of vascular bone, and an inner layer of lamellar bone. Because the outermost layer was dentine, the earliest Ostracoderms were size-limited and could not continue to grow after undergoing metamorphosis [43]. As bony fish with phosphate-based endoskeletons began to evolve, the previously observed bony exoskeletons began breaking into scales instead of large plates, with only the bony plates covering the head retained. These scales and cranial plates likely served as external protection from other creatures [47]. The dissolution of the external skeleton and development of an internal skeletal frame allowed bony fish to continue to grow in size, unhampered by the confines of external mineral secretion and deposition (Figure 9). However, this does not diminish the fact that during this move towards the development of an endoskeleton, other organisms showed a tendency to evolve away from bone. For example, modern lungfish are observed to have very little bony structure [48].

\section{Conclusion}

A primary question surrounding this complex structure of the skeleton concerns the opportunities and pressures that led to the dependence on calcium phosphate rather than calcium carbonate as a building block. While these changes occurred nearly half a billion years ago, the evolving understanding of it is still relevant today. Chemical adaptations are influenced by selective advantages such as 1) The need for storage of energy metabolites and 2) The ability to rapidly retrieve these metabolites with relative celerity. In addition to the need for storage of energy metabolites and the need for increased vascularity to rapidly access metabolites, further implications include the need for a strong support lattice for active muscles and the ability to buffer lactic acid interactions. While evolution is complex, it seems necessary to begin with a framework starting with physiological principles that could be used to describe the importance of calcium phosphate. This paper describes a starting scaffold of the possibilities for understanding bone and its unique qualities for storage, resilience, and adaptability to change. Such reviews bring more people to biological concepts and create memorable experiences that can engage them with their understanding. This could lead to interest among clinicians in furthering discourse and treatment of bone-related diseases, as well as provide the scientific backdrop of the role of phosphate in various bone physiologies. The unique and fascinating characteristics of calcium phosphate are a continued mystery and require much more investigation and research. We hope this review and discussion will motivate continued investigation of the change from calcium carbonate to calcium phosphate in skeletal structures.

\section{Acknowledgement}

This article is dedicated to the memory of our colleague, Dr. Farid Ramji, a pediatric physician, and an inspiring collaborator and teacher.

\section{References}

1. Doherty Alison, Cameron Ghalambor, Seth Donahue (2015) Evolutionary physiology of bone: Bone metabolism in changing environments. Physiology 30: 17-29.

2. Feng Xu, Jay M McDonald (2011) Disorders of bone remodeling. Annu Rev Pathol 6: 121-145.

3. Michigami Toshimi (2019) Skeletal mineralization: Mechanisms and diseases. Ann Pediatr Endocrinol Metab 24: 213-219.

4. Bartram Joe (2014) Let's talk about phosphorus depletion. Oxford University, Environmental Research Doctoral Training Partnership, DTP.

5. (2017) Information about the Cambrian Period. National Geographic.

6. Crook T (1924) The origin of limestones. Nature 114: 733-734.

7. Wagner $D$, Aspenberg $P$ (2011) Where did bone come from? Acts Orthopaedica 82: 393-398.

8. Berrill NJ (1955) The origin of vertebrates. Clarendon, Oxford, U.K.

9. Cao-Pham AH, Hiong KC, Boo MV, et al. (2019) Calcium absorption in the fluted giant clam, Tridacna squamosa, may involve a homolog of voltage-gated calcium channel subunit $\alpha 1$ (CACNA1) that has an apical localization and displays light-enhanced protein expression in the ctenidium. J Comp Physiol B 189: 693-706.

10. Brady J, Hollum J (2000) Descriptive chemistry of the elements. John Wiley and Sons, New York, 5-16, 29-35, 53-61.

11. Hsia Connie CW, Schmitz A, Lambertz M, et al. (2013) Evolution of air breathing: Oxygen homeostasis and the transitions from water to land and sky. Compr Physiol 3: 849-915.

12. Melanie L, Lewis JP (2016) Oxygen isotopes in Molluscan shell: Applications in environmental archaeology. Environmental Archaeology 21: 295-306.

13. Tony $H$ (2012) Why nature chose phosphate to modify proteins. Philos Trans R Soc Lond B Biol Sci 367: 2513-2516.

14. Elijah A (1978) Invertebrate collagens. Science 202: 591-598.

15. Levine M (2011) Fibrillar and non-fibrillar collagens and integrins. Topics in Dental Biochemistry. Springer, Berlin, Heidelberg.

16. Mark T, Snaddon JL, Turner EC (2017) Scientific research on animal biodiversity is systematically biased towards vertebrates and temperate regions. PLoS One 12.

17. Gheorghe T, Pasca R-D (2015) On the collagen mineralization. A review. Clujul Medical 88: 15-22.

18. Goretti Penido M, Alon US (2012) Phosphate homeostasis and its role in bone health. Pediatr Nephrol 27: 2039-2048.

19. Moss ML (1963) The biology of acellular teleost bone. Ann N Y Acad Sci 109: 337-350.

20. Marny MA (2008) An overview of bone cells and their regulating factors of differentiation. Malays J Med Sci 15: 4-12.

21. Shahar R, Dean M (2012) The structure-mechanics relationship and the response to load of the acellular bone of neoteleost fish: A review. J Appl Ichthyol 28: 320-329.

22. Ron S, Dean MN (2013) The enigmas of bone without osteocytes. BoneKEy Reports 2: 343.

23. Structure and composition of bone. University of Cambridge. 
Citation: Wu DH, Preskitt C, Gresham-Fiegel C (2021) Chemical and Physiological Change from Calcium Carbonate to Calcium Phosphate in Skeletal Structures. Insights Biomed Res 5(1):139-148

24. Holland HD (1984) The chemical evolution of the atmosphere and oceans. Princeton Univ Press, Princeton, NJ.

25. Benton S, Aflalo E, Tynyakov J, et al. (2016) Calcium phosphate mineralization is widely applied in crustacean mandibles. Scientific Reports 6.

26. Trofimov AD, Ivanova AA, Zyuzin MV, et al. (2018) Porous inorganic carriers based on silica, calcium carbonate and calcium phosphate for controlled/modulated drug delivery: Fresh outlook and future perspectives. Pharmaceutics 10: 167.

27. Wiechers HNS, P Sturrock, Marais GR (1975) Calcium carbonate crystallization kinetics. Water Research 9: 835-845.

28. Addadi L, Raz S, Weiner S (2003) Taking advantage of disorder: Amorphous calcium carbonate and its roles in biomineralization. Advanced Materials 15: 959-970.

29. Albéric M, Bertinetti L, Zou Z, et al. (2018) The crystallization of amorphous calcium carbonate is kinetically governed by ion impurities and water. Adv Sci 5.

30. Horne $F$ (2006) How are seashells created? Or any other shell, such as a snail's or a turtle's? Scientificamerican.

31. Szpak P (2011) Fish bone chemistry and ultrastructure: Implications for taphonomy and stable isotope analysis. Journal of $\mathrm{Ar}$ chaeological Science 38: 3358-3372.

32. Carstomn S, Epple M (2003) Carbonated calcium phosphates are suitable $\mathrm{pH}$-stabilising fillers for biodegradable polyesters. Biomaterials 24: 2037-2043.

33. Understanding Porosity and Density. Wisconsin Geological Natural History Survey.

34. Kumar S, Hedges S (1998) A molecular timescale for vertebrate evolution. Nature 392: 917-920.

35. Kutchera U, Niklas K (2004) The modern theory of biological evolution: An expanded synthesis. Naturwissenschaften 91: 255276.

36. St-Onge P, Miron G, Moreau G (2007) Burrowing behaviour of the softshell clam (Mya arenaria) following erosion and transport. Journal of Experimental Marine Biology and Ecology 340: 103-111.
37. Koehl M, Quillin KJ, Pell CA (2000) Mechanical design of fiber-wound hydraulic skeletons: The stiffening and straightening of embryonic notochords. American Zoology 40: 28-41.

38. Ruben J, Bennett A (1980) Antiquity of the vertebrate pattern of activity metabolism and its possible relation to vertebrate origins. Nature 286: 886-888.

39. Ruben J, Bennett A (1987) The evolution of bone. Evolution 41: 1187-1197.

40. Hannink G, Arts JC (2011) Bioresorbability, porosity and mechanical strength of bone substitutes: What is optimal for bone regeneration? Injury 42: 22-25.

41. Cooper D, Kawalilak C, Harrison K, et al. (2016) Cortical bone porosity: What is it, why is it important, and how can we detect it? Curr Osteoporos Rep 14: 187-198.

42. Hirasawa T, Kuratani S (2015) Evolution of the vertebrate skeleton: Morphology, embryology, and development. Zoological Lett 1: 2.

43. Orkin P (1952) Bone as a skeletal structure and as a mineral reserve. Proceedings of the Nutrition Society, University of St Andrews.

44. Institute of Medicine (US) Standing Committee on the Scientific Evaluation of Dietary Reference Intakes (1997) 4, Calcium. Dietary reference intakes for calcium, phosphorus, magnesium, vitamin D, and Fluoride. National Academies Press (US), Washington (DC).

45. Full R, Herreid C (1984) Fiddler crab exercise: The energetic cost of running sideways. J Exp Biol 109: 141-161.

46. Margolis HC, Moreno EC (1992) Kinetics of hydroxyapatite dissolution in acetic, lactic, and phosphoric acid solutions. Calcif Tissue Int 50: 137-143.

47. Kuratani S (2004) Evolution of the vertebrate jaw: comparative embryology and molecular developmental biology reveal the factors behind evolutionary novelty. J Anat 205: 335-347.

48. Bentov S, Aflalo E, Tynyakov J, et al. (2016) Calcium phosphate mineralization is widely applied in crustacean mandibles. Sci Rep 6.

49. Nield D (2017) The evidence is building that our teeth evolved from fish skin. Nature.

DOI: $10.36959 / 584 / 460$

Copyright: (c) $2021 \mathrm{Wu} \mathrm{DH}$, et al. This is an open-access article distributed under the terms of the Creative Commons Attribution License, which permits unrestricted use, distribution, and reproduction in any medium, provided the original author and source are credited. 